\title{
Validation of the modified Arabic version of Activities Specific Balance Confidence Scale (ABC) among Egyptian patients with vestibular disorders
}

\author{
Original \\ Hossam Sanyelbhaa Talaat ${ }^{1}$, Ahmed Mahmoud Zein El Abedein ${ }^{2}$ and Fatma Shaaban \\ Article \\ Abd Elkawy \\ ${ }^{I}$ Professor and Head of Audiology Unit, Faculty of medicine, Menoufia University, ${ }^{2} M D$ of \\ audiology, Faculty of medicine, Menoufia University, ${ }^{3} \mathrm{MBBCH}$, Faculty of medicine, Ain \\ Shams University
}

\begin{abstract}
Background: The $\mathrm{ABC}$ scale is a questionnaire to evaluate the risk of fall in people with imbalance.

Objectives: Modify and validate the Arabic form of the ABC scale on the Egyptians with vestibular disorders.

Methods: The Arabic version of the $\mathrm{ABC}$ scale has been revised and modified to replace the words that is ambiguous on the Egyptians. The revised version has been back translated and judged by expert committee to confirm its matching with the original English version. The final form is applied to the control group which is composed of 100 healthy Egyptians with age (18 - 45) years and a study group composed of 60 Egyptian patients having vestibular disorders (VD) that match the control group in age and sex.

Results: The results gave excellent reliability with Cronbach's alpha (0.94) among patients and (0.75) among controls and the corrected item-total correlation $>0.3$ for all items. The repeatability was found high for control and VD group. The total scores for VD patients were significantly different from the controls.

Conclusion: The modified Arabic ABC scale is reliable and valid subjective tool in the assessment and follow up of VD among Egyptians.
\end{abstract}

Key Words: Arabic, ABC scale, Egyptians

Received: 14 February 2019, Accepted: 18 May 2019

Corresponding Author: Ahmed Mahmoud Zein El Abedein, MD, Department of Audiology, Faculty of medicine, Menoufia Universitya, Egypt, Tel.: +2 01090027979, E-mail: am_zein14@yahoo.com

ISSN: 2090-0740, July 2019 Vol.20, No.2

\section{INTRODUCTION:}

The vestibular system plays an important role in controlling the body balance therefore; any insult of the vestibular system impaire balance and increase the risk of falling ${ }^{[3]}$. Having the ability to assess patient's functional balance and identify the activities and situations that increase their risk for falling is necessary to prevent falls. Therefore, instruments have been designed and used to evaluate the body balance and balance confidence in persons with vestibular disorders like the Activities Specific balance Confidence Scale ${ }^{[2]}$. VD patients are 12 times more prone to falling attacks ${ }^{[12]}$. Certain daily activities was avoided by Patients having vestibular disorders because of their fear of falling ${ }^{[5]}$. Assesment of the patient functional balance ability during his daily life activities is important to avoid falling in the future. That's why a lot of assessment tools have been developed to evaluate the patients balance one of them is the ABC scale ${ }^{[8]}$. The activities specific balance confidence scale is popular, reliable and valid tool for evaluation of the functional balance and balance confidence. It is a subjective measure of confidence in the performance of various activities without falling or sense of unsteadiness ${ }^{[7]}$.The $\mathrm{ABC}$ was originally established to be used with patients having functional balance disability like with vestibular disorders and its consisted of 16 items with a total score between 0 and 100, where higher scores equate to higher balance confi $\neg$ dence and vice versa ${ }^{[9]}$.

\section{METHODS:}

The approval was taken to progress in this study from the ethical committee in the Menoufia University and the patients collected from the audio-vestibular medical unit in Menoufia university in Egypt in time range from October 2017 till September 2018. The persons in this study were Arabic language speakers all of them were native Arabic who were capable of speaking and understanding the Arabic language. They were divided into two groups with the following criteria:

The control group composed of 100 healthy Egyptian adult persons who are in age range from 18 to 45 years, didn't complain of dizziness, neurological or labyrinthine diseases. The vestibular disorders group consisted of 60 vestibular disorders patients according to the clinical 
assessment by the bedside tests and VNG in audiovestibular clinic of the Menoufia university hospital. Any patient with dizziness due to systemic diseases like: cardiopulmonary disorder, thyroid dysfuction or anemia was excluded and also all patients with musculoskeletal, Mental or psychiatric disorders.

Firstly, the license to modify the Arabic ABC scale was obtained from the professor Aliaa Alghwiri who translated the original $\mathrm{ABC}$ scale from the authors professors Powell and Myers ${ }^{[10]}$, which was used in this study for modifications. This questionnaire is consisted of 16 items with a total score that ranges between 0 and 100 .

The 1st step was modification of the previously translated Arabic version of the $\mathrm{ABC}$ scale (a new forward translation) to be suitable with the general Egyptian language. The revised version of the scale has been back translated and judged by expert committee to confirm its matching with the original English version in accordance with the World Health Organization (WHO) forward/backward translation protocol for the translation of the evaluation tools ${ }^{[11]}$. A review by the four translators with the authors to make a comparison between the new forward translations with a backward translations to get the convenient terms and make a pre-final model of the modified Arabic ABC scale.

Then, the step of pre-testing for the final version of the modified scale: by using a pilot test on 10 patients and this is to check for any errors and/or any strange or difficult word and a final version of the modified Arabic ABC scale was ready after getting the patients' feedback of the pilot test.

The modified Arabic version of the ABC scale was introduced and applied on the persons of both groups 'the control and VD groups' after taking their informed consent for the participation in this study. The total scores of the modified Arabic ABC scale is calculated by the division of the total itmes sum ${ }^{[16]}$

\section{The Statistical analysis}

In the current study results were statistically analyzed by SPSS version 22 (SPSS Inc., Chicago, IL, USA) and the sample adequacy was detected by statisticians. The quantitative data is presented in the form of ( standard deviation and mean) the differences in results are considered to be significant if the $\mathrm{p}$ values were $<0.05$. The modified Arabic ABC scale was tested for its validity and reliability. The reliability of the scale is measured by internal consistency ( using Cronbach's alpha factor) the test is being reliable when alpha is $>0.7$ and by test re-test consistency ( using Spearman's correlation coefficient). The Reciver Operating Characteristics curve (ROC) was used to find the optimal cut off value for detecting the probable risk of falling in VD group. The Specificity, Sensitivity, Positive predictive values and Negative predictive values were tested for possible cut off values.

\section{RESULTS:}

The control group composed of 100 persons 62 females and 38 males, with mean age $32.7 \pm 6.60$ years. The age range between 18 and 45 years. The VD group consisted of 60 patients with 24 males and 36 females, with mean age $34.7 \pm 7.49$ years, (the range of age from 18 to 45 years) the two groups show non-significant statistical difference between them as regarding the age and sex $(\mathrm{P}>0.05)$ using t-test and chi-square test respectively. As regard correlation between the age and total scores there was a statistically significant negative correlation between age and $\mathrm{ABC}$ scale total scores of cases and control groups in the 1 st and 2 nd visits $\mathrm{p}<0.001$ as shown by using the Pearsons' correlation test $(\mathrm{r}=-0.44)$.

Diagnosis of the dizziness cause in patients in the VD group was; Meniere's disease in $16 / 60$ patients $(26.6 \%)$, Benign Paroxysmal Positional vertigo in 14/60 (23.3\%), migraine in $14 / 60$ patients $(23.3 \%)$, vestibular neuritis in $6 / 60$ patients $(10 \%)$, vertigo of unknown cause in $6 / 60$ $(10 \%)$ and central vestibular disorders in 4/60 (6.6\%).

Reliability of the modified Arabic ABC scale was tested using the test re-test reliability \& internal consistency. All of the 60 participants of this study completed the scale twice. The total score for the first visit is ranged from 33.7 to 94.3 with mean $=62.8$ and $\mathrm{SD}=21.3$ and for the 2 nd visit total scores ranged from 33.13 to 92.5 with mean $=$ 65.6 and $\mathrm{SD}=19.5$.

The scale reflected good agreement to the test re-test values for all items of the modified Arabic form of the $\mathrm{ABC}$ scale indicating a good repeatability among patients group with spearman correlation factor $=0.97$ between the 1st and 2nd visit and CI 95\% $=(0.92-0.98)$ with $\mathrm{P}<0.001$ which is highly significant which demonstrated in (Fig.1). The mean score of the scale at the item level ranged from 40.3 for "walk on icy sidewalks" to 76.6 for "walk around the house". The test-retest reliability of the scale item score reflected high agreement with a range of CI 95\% from 0.67- 0.91 for "walk up and down stairs" to $0.96-0.98$ for "walk in a crowd or get bumped" as shown in (Table 1). 


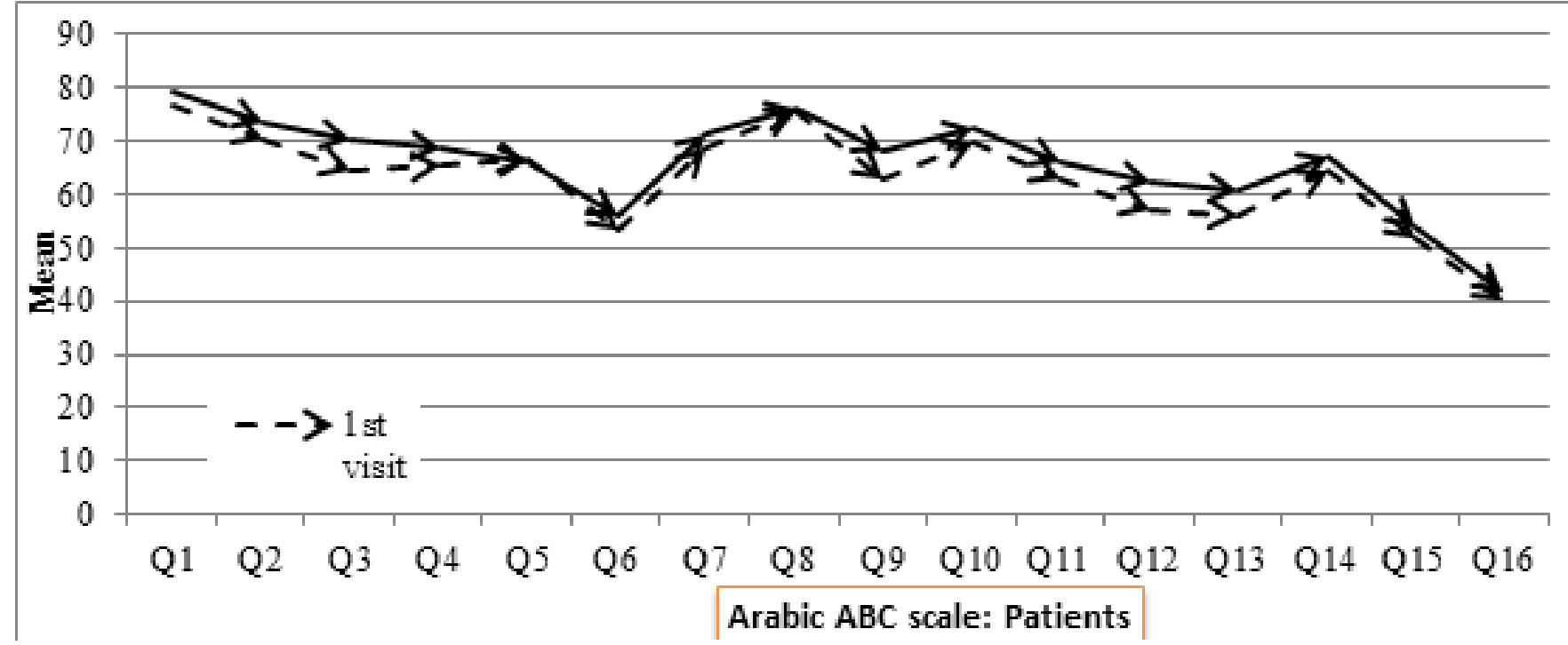

Fig. 1: Test re-test reliability descriptive statistics and repeatability measures of the modified Arabic ABC scale in the 1st and 2nd visit among patients.

Table 1: This table shows that there is good agreement of the test re-test values of all items of the Arabic form of ABC scale indicating good repeatability among patients $(\mathrm{P}<0.001)$.

\begin{tabular}{ccccc}
\hline \multirow{2}{*}{ ABC scale } & 1st visit & 2nd visit & Spearman correlation & CI 95\% \\
\cline { 2 - 3 } Q 1 & $76.66 \pm 25.55$ & Mean \pm SD & $0.881^{*}$ & $0.781-0.941$ \\
Q 2 & $70.33 \pm 30.64$ & $73.0 \pm 20.39$ & $0.823^{*}$ & $0.674-0.918$ \\
Q 3 & $64.33 \pm 29.07$ & $70.0 \pm 26.16$ & $0.958^{*}$ & $0.916-0.983$ \\
Q 4 & $65.66 \pm 28.72$ & $68.66 \pm 26.39$ & $0.957^{*}$ & $0.915-0.977$ \\
Q 5 & $66.66 \pm 27.59$ & $66.0 \pm 23.80$ & $0.924^{*}$ & $0.858-0.958$ \\
Q 6 & $53.33 \pm 29.38$ & $55.66 \pm 25.86$ & $0.955^{*}$ & $0.917-0.974$ \\
Q 7 & $68.66 \pm 31.75$ & $71.0 \pm 28.32$ & $0.863^{*}$ & $0.752-0.934$ \\
Q 8 & $75.66 \pm 28.36$ & $76.0 \pm 23.08$ & $0.849^{*}$ & $0.726-0.922$ \\
Q 9 & $62.66 \pm 30.46$ & $68.0 \pm 26.47$ & $0.952^{*}$ & $0.895-0.980$ \\
Q 10 & $69.66 \pm 31.40$ & $72.33 \pm 26.89$ & $0.860^{*}$ & $0.793-0.935$ \\
Q 11 & $63.0 \pm 26.82$ & $66.0 \pm 22.33$ & $0.926^{*}$ & $0.865-0.961$ \\
Q 12 & $57.0 \pm 28.54$ & $62.0 \pm 26.21$ & $0.943 *$ & $0.894-0.975$ \\
Q 13 & $56.0 \pm 26.50$ & $60.66 \pm 28.86$ & $0.979^{*}$ & $0.960-0.989$ \\
Q 14 & $64.33 \pm 31.74$ & $66.66 \pm 29.49$ & $0.899^{*}$ & $0.813-0.948$ \\
Q 15 & $52.0 \pm 25.95$ & $53.66 \pm 26.48$ & 0.939 & $0.890-0.960$ \\
Q 16 & $40.33 \pm 24.76$ & $41.66 \pm 26.04$ & $0.920^{*}$ & $0.872-0.945$ \\
\hline Total score & $62.89 \pm 21.31$ & $65.64 \pm 19.55$ & $0.970^{*}$ & $0.923-0.989$ \\
\hline
\end{tabular}


The internal consistency of the test is measured by Cronbach's Alpha factor, It helps in deciding if different items in the scale were measuring the same underlying concept. The internal consistency of the modified Arabic $\mathrm{ABC}$ scale was tested by using the Cronbach's Alpha ( Cronbach's Alpha $=0.94$ in the VD group \& $=0.75$ in controls ). That's mean the modified Arabic ABC scale has a good internal consistency indicating excellent test reliability and strong test internal consistency as Cronbach's alpha with 0.946 among patients and Cronbach's alpha with 0.756 among controls and all items have the corrected item total correlation $>0.3$ as shown in (Table 2).
As regarding the validity of the modified Arabic $\mathrm{ABC}$ scale it showed that AUC (Area Under the Curve) is 0.96 to predict the risk of falling in patients with vestibular disorders. Values higher than 0.7 or equal would be considered a strong predictors. The cut-off point 80.94 had the highest sensitivity which is $93.0 \%$ and specificity $100 \%$.Asignificant large correlation between the scores of the scale in the first trial between cases and control. The Receiver operating characteristics (ROC) curve for using Arabic form of $\mathrm{ABC}$ scale to predict risk of falling among patients with vestibular disorders. The ROC curve is created by plotting the true positive rate $(\mathrm{TPR})=$ (sensitivity) against the false positive rate $(\mathrm{FPR})=(1-$ specificity $)$ at various threshold settings demonstrated at (Fig. 2).

Table 2: This table shows that the modified Arabic form of the activities specific balance confidence scale has excellent reliability and strong internal consistency as Cronbach's alpha with 0.946 among patients and Cronbach's alpha with 0.756 among controls and all items have corrected item-total correlation more than 0.3 .

\begin{tabular}{ccccc}
\hline & \multicolumn{4}{c}{ Internal consistency of Arabic ABC scale } \\
\cline { 2 - 5 } ABC scale & \multicolumn{2}{c}{ Patients } & Controls \\
\cline { 2 - 5 } & $\begin{array}{c}\text { Corrected Item- } \\
\text { Total Correlation }\end{array}$ & $\begin{array}{c}\text { Cronbach's Alpha } \\
\text { if item Deleted }\end{array}$ & $\begin{array}{c}\text { Corrected Item- } \\
\text { Total Correlation }\end{array}$ & $\begin{array}{c}\text { Cronbach's Alpha } \\
\text { if item Deleted }\end{array}$ \\
\hline Q 1 & 0.488 & 0.947 & 0.305 & 0.754 \\
Q 2 & 0.734 & 0.942 & 0.331 & 0.746 \\
Q 3 & 0.605 & 0.945 & 0.391 & 0.742 \\
Q 4 & 0.692 & 0.943 & 0.309 & 0.747 \\
Q 5 & 0.481 & 0.947 & 0.356 & 0.743 \\
Q 6 & 0.620 & 0.945 & 0.505 & 0.728 \\
Q 7 & 0.719 & 0.942 & 0.343 & 0.752 \\
Q 8 & 0.816 & 0.940 & 0.378 & 0.746 \\
Q 9 & 0.751 & 0.942 & 0.338 & 0.735 \\
Q 10 & 0.891 & 0.938 & 0.518 & 0.730 \\
Q 11 & 0.618 & 0.944 & 0.497 & 0.738 \\
Q 12 & 0.814 & 0.940 & 0.405 & 0.729 \\
Q 13 & 0.866 & 0.939 & 0.509 & 0.750 \\
Q 14 & 0.855 & 0.939 & 0.297 & 0.745 \\
Q 15 & 0.695 & 0.943 & 0.343 & 0.763 \\
Q 16 & 0.582 & 0.945 & 0.344 & 0.756 \\
\hline Cronbach's alpha & & 0.946 & &
\end{tabular}

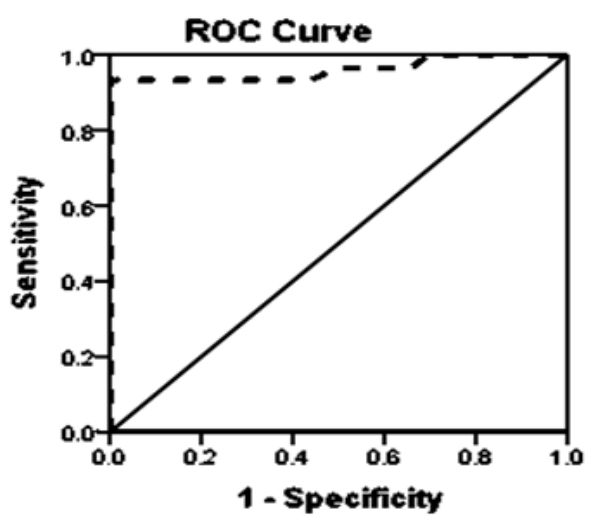

Fig. 2: The ROC curve to predict risk of falling among patients with vestibular disorders. The it is created by plotting the true positive rate $(\mathrm{TPR})=($ sensitivity $)$ against the false positive rate $(\mathrm{FPR})=(1$ - specificity $)$ at various threshold settings.

\section{DISCUSSION:}

The purpose of this study was to examine the reliability and validity of the modified Arabic version of the $\mathrm{ABC}$ scale among sample of the Egyptian population with vestibular disorder. The Arabic version of the scale underwent some modifications in its structural words to be convenient with the Egyptian people to be easily formed. Findings of this study demonstrated a high degree of intra-rater reliability and high test re-test reliability of the modified Arabic scale. The reliability results obtained in this study for the modified Arabic version of the scale showed results close to those obtained for the original English version ${ }^{[10]}$.

In a recent study $\mathrm{ABC}$ scale was found to be responsive 
in persons with vestibular disorders ${ }^{[4]}$. According to Myers et al., the $\mathrm{ABC}$ score may help in classifying patients into one of 3 levels of physical functioning : low level $(\mathrm{ABC}<50)$, moderate level $(<50 \mathrm{ABC}<80)$, and high level of physical functioning $(\mathrm{ABC}>80)^{[1]}$.

Therefore, in this study, a mean score of 63 on the modified Arabic version of the ABC scale would classify our participant as being at a moderate level of functioning ${ }^{[6]}$. similar findings were demonstrated in a study conducted by Alghwiri et al. (2016) on 82 patients with vestibular disorders with mean age 43 (between 29 and 57 years old) female predominance. Their study results were similar to the present study in reliability and validity.

\section{CONCLUSION:}

Modification of the Arabic version of the ABC scale was developed and was found to be a valid and reliable tool with Egyptian patients having vestibular disorders. The modified Arabic version of the ABC scale can be used in assessment of risk of falling in patients with vestibular disorders.

\section{DISCLOSURE OF INTEREST:}

The authors declare that they don't have conflicts of interest concerning this article.

\section{ACKNOWLEDGMENT:}

special thanks to professor Aliaa A. Alghwiri in the Department of Physical Therapy, The Faculty of Rehabilitation Sciences, in University of Jordan, Amman, Jordan who gave me the original Arabic scale and gave me the acceptance to use her Arabic version in my research and to the department of Otorhinolaryngology/ Head and Neck surgery, Menoufia University for financial support.

\section{REFERENCES:}

1. A.M. Myers, P.C. Fletcher, A.H. Myers and . Sherk, Discriminitive and evaluative properties of the activities- specific balance confidence(ABC) scale. JGerontol A BIOL Sci Med Sci(1998). 53,M287-294.

2. G.P.jacobson and C.W. Newman, The development of the Dizziness Handicap Inventory, Arch Otolaryngol Head Neck Surg (1990). 116, 424-427.
3. K.Gill-Body, M. Beninatoand K. D., Relationship among balance impairmments, functional performance, and disability in people with peripheral vestibular hypofunction, Phys Ther (2000): 80, 748-758.

4. L.A. Friscia, M.T. Morgan, P.J Sparto, J.M. Furman and S.L. Whitney Responsivness of self-report measures in individuals with vertigo, dizziness, and unsteadiness, otol neurotol (2014). $35,884-888$.

5. L.Yardley, B. Dibb and G. Osborne. Factors associated with ing recovery from balance disorders. JAnxiety Disord15 quality of life in Meniere's disease, ClinOtolaryngol Allied, (2001): 107-119.

6. M.K. Mak and M.Y. Pang, Balance confidence and functional mobility are independently associated with falls in people with Parkinsons's disease, J Neurol (2009). 256, 742-749.

7. Moiz J A, Bansal V, Noohu M M, Gaur S N, Hussain M E. et al. Cross-Cultural Adaptation and Psychometric Analysis of the HindiTranslated Activities-Specific Balance Confidence Scale, Middle East J Rehabil Health Stud. ( 2016).;3(1):e34886.

8. P.C. Hsu and W.C. Miller. Reliability of the Chinese version of the Activities-specific Balance Confidence Scale, Disability Rehabil 28, (2006). $1287-1292$

9. powell LE, Myers AM., The Activities-specific Balance Confidence (ABC) scale 1,Jgerontol A Biol Sci Med Sci , (1995). vol. 50A (pg. M28-34).

10. Powell, L. and A. Myers, The activities-specific balance confidence (ABC) scale. J Gerontol Med Sci 1995: p. M28-M34.

11. World Health Organization. Process of translation and adaptation of instruments.cited 2013, Available from: http; // www.

12. Y. Agrawal, J. Carey, C. Della Santina, M. Schubert and L. Minor 2001-2004, Disorders of balance and vestibular function in adults: Data from the National Health and Nutrition Examination Survey, Arch Intern Med 169 (2009), 938-944. 